\title{
SIGNAL-NOISE IDENTIFICATION OF MAGNETOTELLURIC SIGNALS USING FRACTAL-ENTROPY AND CLUSTERING ALGORITHM FOR TARGETED DE-NOISING
}

\author{
JIN LI, $, \&,, \|$ XIAN ZHANG,${ }^{*}$ JINZHE GONG,${ }^{\dagger}$ JINGTIAN TANG $,{ }^{\ddagger}, \boldsymbol{q}, \|$ \\ ZHENGYONG REN ${ }^{\ddagger}$ GUANG LI, ${ }^{\ddagger}$ YANLI DENG, ${ }^{*}$ and JIN CAI* \\ *Institute of Physics and Information Science \\ Hunan Normal University, Changsha 410081, P. R. China \\ ${ }^{\dagger}$ School of Civil, Environmental and Mining Engineering \\ University of Adelaide, SA 5005, Australia \\ ${ }^{\ddagger}$ School of Geosciences and Info-Physics \\ Key Laboratory of Metallogenic Prediction of Non-Ferrous Metals \\ and Geological Environment Monitor, Ministry of Education \\ Central South University, Changsha 410083, P. R. China \\ §geologylj@163.com \\ 『jttang@mail.csu.edu.cn
}

Received December 20, 2017

Accepted January 21, 2018

Published March 8, 2018

\begin{abstract}
A new technique is proposed for signal-noise identification and targeted de-noising of Magnetotelluric (MT) signals. This method is based on fractal-entropy and clustering algorithm, which automatically identifies signal sections corrupted by common interference (square, triangle and pulse waves), enabling targeted de-noising and preventing the loss of useful information in filtering. To implement the technique, four characteristic parameters - fractal box
\end{abstract}

\footnotetext{
" Corresponding authors.
}

This is an Open Access article published by World Scientific Publishing Company. It is distributed under the terms of the Creative Commons Attribution 4.0 (CC-BY) License. Further distribution of this work is permitted, provided the original work is properly cited. 
dimension (FBD), higuchi fractal dimension (HFD), fuzzy entropy (FuEn) and approximate entropy (ApEn) - are extracted from MT time-series. The fuzzy c-means (FCM) clustering technique is used to analyze the characteristic parameters and automatically distinguish signals with strong interference from the rest. The wavelet threshold (WT) de-noising method is used only to suppress the identified strong interference in selected signal sections. The technique is validated through signal samples with known interference, before being applied to a set of field measured MT/Audio Magnetotelluric (AMT) data. Compared with the conventional denoising strategy that blindly applies the filter to the overall dataset, the proposed method can automatically identify and purposefully suppress the intermittent interference in the MT/AMT signal. The resulted apparent resistivity-phase curve is more continuous and smooth, and the slow-change trend in the low-frequency range is more precisely reserved. Moreover, the characteristic of the target-filtered MT/AMT signal is close to the essential characteristic of the natural field, and the result more accurately reflects the inherent electrical structure information of the measured site.

Keywords: Magnetotelluric; Fractal-Entropy; Clustering; Signal-Noise Identification; DeNoising.

\section{INTRODUCTION}

Magnetotelluric (MT) is an electromagnetic-based exploration method, which measures the orthogonal electric and magnetic fields of the earth surface for identifying the distribution of underground geoelectric structure ${ }^{1 / 2}$ In addition to the impact of terrain, the signal of natural fields is extremely weak and has a wide frequency bandwidth, which leads to the fact that the measured MT and Audio Magnetotelluric (AMT) data are usually corrupted by noise from machineries that emit electromagnetic signals. $\stackrel{3}{3}$ Effective identification and suppression of strong interference from the measured MT/AMT data is essential for a reliable geophysical interpretation. A wide variety of modern signal processing methods have been used for de-noising of MT/AMT data, such as the Hilbert-Huang transform,, 4 variance-ratio wiener filtering,,$[5]$ morphological filtering, 6 the signal subspace enhancement technique ${ }^{7]}$ and the wavelet transform. $\left[{ }^{8}\right.$ These methods attempt to improve the quality of MT/AMT data, which have played a positive role in enhancing the accuracy of the geoelectric structure information extracted from the measured MT/AMT data. However, a common limitation of conventional methods is that the de-noising filter is applied to the whole signal, leading to the loss of useful information ("over-processing") in signal sections without strong interference.

Recent development in signal processing has provided opportunities to improve the noise identification and reduction in MT/AMT data. Fractal analysis is an effective method of analyzing complex nonlinear signals based on the nonlinear system theory. This technique can quantitatively describe the complexity and irregularity of signal morphology 910 Entropy is usually used to represent the degree of disorder of systems and the degree of complexity of signals.11 Fractal-entropy has been used in many fields including image signal processing, ${ }^{12}$ seismic source characterization 13 and porous media analysis.14] 16 Previous studies led by the first author have demonstrated that the morphological fractal dimension and morphological spectral entropy can be used to identify strong interference in MT signals, $\frac{17}{\sqrt{17}}$ however, the identification lacks robustness, and the threshold cannot be automatically obtained for various measurement sites, which can lead to the misjudgment of the boundary of the MT/AMT signal and the interference.

In this paper, a new technique is proposed for automated signal-noise identification and targeted de-noising of MT/AMT signals. A fractal-entropy analysis technique is developed to preprocess MT/AMT signals before an automated classification and a selective use of the wavelet threshold (WT) de-noising technique. The fractal box dimension (FBD), the Higuchi fractal dimension (HFD), the fuzzy entropy (FuEn) and the approximate entropy (ApEn) are extracted from the time-series of measured MT/AMT data as the characteristic parameters. The fuzzy c-means (FCM) clustering method, $18[19$ a multivariate statistical method for studying the classification problem, is used to automatically distinguish signals with strong interference from the rest. The WT de-noising method is used to suppress the identified interference in selected signal sections. The proposed technique is 
validated using signal samples with little noise and those with known interference. The result confirms that the selected four characteristic parameters are suitable to represent the feature of the common interference of square, triangle and pulse waves, enabling automated and robust characterization using the fuzzy c-means clustering algorithm. The technique is applied to a set of field measured MT/AMT data for further validation. Compared with the conventional WT-based overall de-noising method, the proposed technique effectively reserves the slow-change characteristics of the signal in the low-frequency range, therefore improving the quality of the apparent resistivity results and enhancing the identification of the ore concentration area. The effectiveness of the proposed technique is also confirmed by the fact that the processed result more realistically reflects the inherent geoelectric structure information of the measured MT/AMT site.

\section{THEORY}

\subsection{Fractal Analysis}

\subsubsection{Fractal box dimension}

The FBD has a certain noise resistance which can extract the fractal feature from the processed signal with different complexity ${ }^{21}[23]$ Assuming $X$ is a not-empty-bounded subset of $R^{n}$, and $N(X, \varepsilon)$ represents the minimum number of the subset that can cover $X$ set with the maximum diameter $\varepsilon$. The FBD of $X$ is defined as follows:

$$
\operatorname{dim}_{B}(X)=\lim _{\varepsilon \rightarrow 0} \frac{\ln N(X, \varepsilon)}{\ln (1 / \varepsilon)} .
$$

Consider a signal $F=\left\{f_{1}, f_{2}, \ldots, f_{Q}\right\} \subset M$, where $M$ is a closed set in the $n$-dimension Euclid space. As the limitation of Eq. (1) cannot be calculated in accordance with the definition, an approximate method is used, in which $\varepsilon$ is defined as a baseline, and it is gradually enlarged to $k \varepsilon$, with $k \in Z^{+}$. The calculation equation is as follows:

$$
\begin{gathered}
P(k \varepsilon)=\sum_{i=1}^{Q / k} \mid \max \left(f_{k(i-1)+1}, f_{k(i-1)+2},\right. \\
\left.\ldots, f_{k(i-1)+k+1}\right) \\
-\min \left(f_{k(i-1)+1}, f_{k(i-1)+2},\right. \\
\left.\left.\ldots, f_{k(i-1)+k+1}\right)\right) \mid
\end{gathered}
$$

where $i=1,2, \ldots, Q / k ; k=1,2, \ldots, Y ; Y \leq N$ and $N$ is the number of sampling points.
The grid count is as follows:

$$
N_{k \varepsilon}=P(k \varepsilon) /(k \varepsilon)+1 ; \quad N_{k \varepsilon}>1 .
$$

The processed signal is in an irregular fractal form without function description, and merely has fractal dimension in its nonscaling range. A better linear relationship between $\lg k \varepsilon$ and $\lg N_{k \varepsilon}$ is defined as a nonscaling range, and the beginning and ending of this nonscaling range are assumed as $y_{1}$ and $y_{2}$, respectively.

$$
\lg N_{k \varepsilon}=a \lg k \varepsilon+b ; \quad y_{1} \leq k \leq y_{2} .
$$

The slope of this line is determined by the least square method, and the expression of FBD is as follows:

$$
d_{B}=\hat{a}=-\frac{\left(y_{2}-y_{1}+1\right)}{\left(\sum \lg k \lg N_{k \varepsilon}-\sum \lg k \sum \lg N_{k \varepsilon}\right)} .
$$

\subsubsection{Higuchi Fractal Dimension}

Higuchi proposed in 1988 an efficient algorithm for measuring the fractal dimension of discrete time sequences ${ }^{24}$ Higuchi's algorithm calculates the fractal dimension directly from time-series. ${ }^{25}$ It is a very simple and useful method for assessment of nonlinearity of the signal without reconstruction of a strange attractor. ${ }^{26}$ Briefly, the processed signal is analyzed in time sequence $x(1), x(2), \ldots, x(N)$ and it is constructed in a new self-similar time-series $X_{k}^{m}$ as follows:

$$
\begin{aligned}
X_{k}^{m}= & \{x(m), x(m+k), x(m+2 k), \ldots, x \\
& \left.\times\left(m+\operatorname{int}\left(\frac{N-m}{k}\right) k\right)\right\},
\end{aligned}
$$

where $k$ and $m$ are integers, and $\operatorname{int}(h)$ is the integer part of the real number $h$. The parameter $k$ indicates the discrete time interval, whereas $m=1,2, \ldots, k$ represents the initial time value. The length $L_{m}(k)$ is computed for each of $k$ timeseries or curves $X_{k}^{m}$.

$$
\begin{aligned}
L_{m}(k)=\frac{1}{k} & {\left[\left(\sum_{i=1}^{\operatorname{int}\left(\frac{N-m}{k}\right)} \mid x(m+i k)\right.\right.} \\
& \left.-x(m+(i-1) k) \mid) \frac{N-1}{\operatorname{int}\left(\frac{N-m}{k}\right) k}\right],
\end{aligned}
$$


where $N$ is the length of the original time-series $X$ and $(N-1) /\{\operatorname{int}[(N-m) / k] k\}$ is a normalization factor. The parameter $L_{m}(k)$ is averaged for all $m$ forming the mean value of the curve length $L(k)$ for each $k=1,2, \ldots, k_{\max }$ as follows:

$$
L(k)=\frac{\sum_{m=1}^{k} L_{m}(k)}{k} .
$$

The HFD is defined as the slope of least square linear best fit from the plot of $\ln (L(k))$ versus $\ln (1 / k)$ :

$$
\mathrm{HFD}=\ln (L(k)) / \ln (1 / k) .
$$

As the parameter $k_{\max }$ is much more dependent, 27 we select $k_{\max }=8$ for processing.

\subsection{Entropy Analysis}

\subsubsection{Fuzzy entropy}

The FuEn is a kind of fuzzy sets, and is used to measure the complexity and irregularity of the concept ${ }^{28}$ It has independence and consistency. The definition of FuEn is explained in this section.

Assuming time-series $u=\{u(1), u(2), \ldots, u(N)\}$ consists a $m$ dimensional vector:

$$
\begin{aligned}
X_{i}^{m}= & \{u(i), u(i+1), \ldots, u(i+m-1)\}-u_{0}(i), \\
& i=1,2, \ldots, N-m+1,
\end{aligned}
$$

where $N$ is the length of time-series, $m$ is the embedding dimension, $X_{i}^{m}$ represents $m$ consecutive $u$ values, commencing with the $i$ th point and generalized by removing their baseline. Then, $u_{0}(i)=\frac{1}{m} \sum_{j=0}^{m-1} u(i+j)$ represents the average of the $m$ values.

Assuming $d_{i j}^{m}$ is denoted as the maximum distance between $X_{i}^{m}$ and $X_{j}^{m}, d_{i j}^{m}$ can be calculated as follows:

$$
\begin{gathered}
d_{i j}^{m}=d\left[X_{i}^{m}, X_{j}^{m}\right]=\max _{k \in(0, m-1)}\left\{\mid u(i+k)-u_{0}(i)\right. \\
\left.-\left[u(j+k)-u_{0}(j)\right] \mid\right\}, \\
\quad i, j=1, \ldots, N-m, \quad i \neq j .
\end{gathered}
$$

Assuming $u\left(d_{i j}^{m}, n, r\right)$ is a fuzzy function, the similarity degree $D_{i j}^{m}$ can be given as follows:

$$
D_{i j}^{m}=u\left(d_{i j}^{m}, n, r\right)=\exp \left(-\left(d_{i j}^{m} / r\right)^{n}\right),
$$

where $n$ is the boundary gradient, $r$ is the boundary width.
Assuming $\phi^{m}(n, r)$ is defined as follows:

$$
\begin{aligned}
\phi^{m}(n, r)= & \frac{1}{N-m} \sum_{i=1}^{N-m} \\
& \times\left(\frac{1}{N-m-1} \sum_{j=1, j \neq i}^{N-m} D_{i j}^{m}\right) .
\end{aligned}
$$

Similarly, repeating the above steps and obtaining a set of the $m+1$ dimensional vectors. The definition of $\phi^{m+1}(n, r)$ is as follows:

$$
\begin{aligned}
\phi^{m+1}(n, r)= & \frac{1}{N-m} \sum_{i=1}^{N-m} \\
& \times\left(\frac{1}{N-m-1} \sum_{j=1, j \neq i}^{N-m} D_{i j}^{m+1}\right) .
\end{aligned}
$$

Finally, according to the above-mentioned steps, the definition of FuEn is as follows:

$$
\begin{aligned}
& \operatorname{FuEn}(m, n, r) \\
& \quad=\lim _{N \rightarrow \infty}\left(\ln \phi^{m}(n, r)-\ln \phi^{m+1}(n, r)\right) .
\end{aligned}
$$

If $N$ is limited, $\operatorname{FuEn}(m, n, r, N)$ can be defined as follows:

$$
\operatorname{FuEn}(m, n, r, N)=\ln \phi^{m}(n, r)-\ln \phi^{m+1}(n, r) .
$$

\subsubsection{Approximate entropy}

The ApEn is a method which can statistically quantify the complexity of time-series based on edge probability distribution. 29 The definition of ApEn is explained in this section.

Given the length of $N$ points original timeseries $u=\{u(1), u(2), \ldots, u(N)\}$, which consists of $m$ dimensional vector: $X(i)=\{x(1), x(2), \ldots$, $x(i+m-1)\} i=N-m+1$.

The distance $d[X(i), X(j)]$ between $X(i)$ and $X(j)$ is defined as follows:

$$
\begin{aligned}
& d[X(i), X(j)] \\
& \quad=\max _{k=1,2, \ldots, m}|x(i+k-1)-x(j+k-1)| .
\end{aligned}
$$

Assuming tolerance window is $r$, count the number of $j(j=1,2, \ldots, N-m+1)$ so that 
$d[X(i), X(j)] \leq r$, denoted as $C^{m}(i)$. Then, for $i=1,2, \ldots, N-m+1, C_{r}^{m}(i)$ is defined as follows:

$$
C_{r}^{m}(i)=C^{m}(i) /(N-m+1) .
$$

Compute the natural logarithm $\varphi^{m}(r)$ of each $C_{r}^{m}(i)$ :

$$
\varphi^{m}(r)=\frac{1}{N-m+1} \sum_{i=1}^{N-m+1} \ln C_{r}^{m}(i) .
$$

Similarly, increase the dimension to $m+1$. Repeat the above steps and get $C_{r}^{m+1}(i)$ and $\varphi^{m+1}(r)$.

The ApEn is defined as follows:

$$
\operatorname{ApEn}(m, r)=\lim _{N \rightarrow \infty}\left[\varphi^{m}(r)-\varphi^{m+1}(r)\right] .
$$

If $N$ is limited, $\operatorname{ApEn}(m, r, N)$ can be defined as follows:

$$
\operatorname{ApEn}(m, r, N)=\varphi^{m}(r)-\varphi^{m+1}(r) .
$$

\subsection{Fuzzy C-Means Clustering}

The FCM clustering is a typical unsupervised fuzzy clustering method, and it is mainly applied in the area of pattern recognition and image segmentation. 30 The FCM algorithm is explained in this section.

Given a limited data set of $X=\left\{x_{1}, x_{2}, \ldots, x_{n}\right\}$, $n$ is the number of samples, the objective is to divide $X$ into $c$ fuzzy type. An objective function is defined as follows:

$$
J(U, V)=\sum_{i=1}^{c} \sum_{j=1}^{n}\left(u_{i j}\right)^{m}\left(d_{i j}\right)^{2},
$$

where $u_{i j}$ represents the membership degree of $i$ th cluster center in the $j$ th classified sample.

The calculation of $u_{i j}$ is as follows:

$$
u_{i j}=\frac{1}{\sum_{k=1}^{c}\left(\frac{d_{i j}}{d_{k j}}\right)^{2 /(m-1)}},
$$

where $m$ is a weighting exponent, $m=[1,+\infty)$. $d_{i j}=\left\|x_{j}-V_{i}\right\|$ is the Euclidean distance between $x_{j}$ and $V_{i} . V=\left(V_{1}, V_{2}, \ldots, V_{i}, \ldots, V_{c}\right)^{T}$ is the cluster center, $V_{i}$ is the $i$ th of cluster center vector. The membership $u_{i j}$ has some constraints, such as $\sum_{i=1}^{c} u_{i j}, j=1,2, \ldots, n, 0<u_{i j}<1$ and $0<\sum_{j=1}^{n} u_{i j}<n, i=1,2, \ldots, c$.

The cluster center $V_{i}$ is calculated as follows:

$$
V_{i}=\frac{\sum_{j=1}^{n} u_{i j}^{m} x_{j}}{\sum_{j=1}^{n} u_{i j}^{m}} .
$$

FCM clustering is an iterative optimization algorithm. According to the membership matrix $u_{i j}$,
FCM clustering can be determined to classify the attribution of object.

\subsection{Wavelet Threshold De-Noising}

Wavelet threshold (WT) de-noising method has many advantages, such as low-entropy, multiresolution, de-correlation and base-selection flexibility ${ }^{31}$ The method includes three steps: Firstly, the original signal is decomposed by wavelet transform, and the coefficients of each scale are obtained. Then, the threshold is processed. Finally, wavelet coefficients are reconstructed to obtain the useful signal after de-noising.

In this paper, we select the soft threshold for denoising. When the absolute value of wavelet coefficient is less than the threshold, $\hat{w}$ is 0 . Otherwise, subtracts the threshold. The calculation formula is defined as follows:

$$
\hat{w}= \begin{cases}\operatorname{sgn}(w)(|w|-\lambda), & |w| \geq \lambda, \\ 0, & |w|<\lambda,\end{cases}
$$

where $w$ and $\hat{w}$ are the wavelet transform coefficients before and after the WT de-noising, respectively. $\operatorname{sgn}()$ is the symbol function; $\lambda$ is the soft threshold, $\lambda=\sigma \sqrt{2 \lg (N)} ; \sigma$ is a noise variance estimate, $\sigma=\frac{\operatorname{median}(|w|)}{0.6745}$, median is the median function. In this paper, we use the wavelet function sym6 with 5 levels to suppress interference.

\section{ALGORITHM ILLUSTRATION AND VERIFICATION}

In this section, the proposed technique is illustrated and validated using data samples with known interference.

\subsection{Feature Extraction}

In order to understand the characteristics of various MT data, a library of 200 data samples is used. The data samples are all from field measurements, and the library includes high-quality MT data (referred as "MT data without interference"), MT data with square wave interference, MT data with triangle wave interference and MT data with pulse wave interference. There are 50 samples for each data type. The high-quality MT data was collected from remote areas with no human activities in Qinghai province, and the three types of strong interference were collected from the Luzong ore concentration area in Anhui province. 

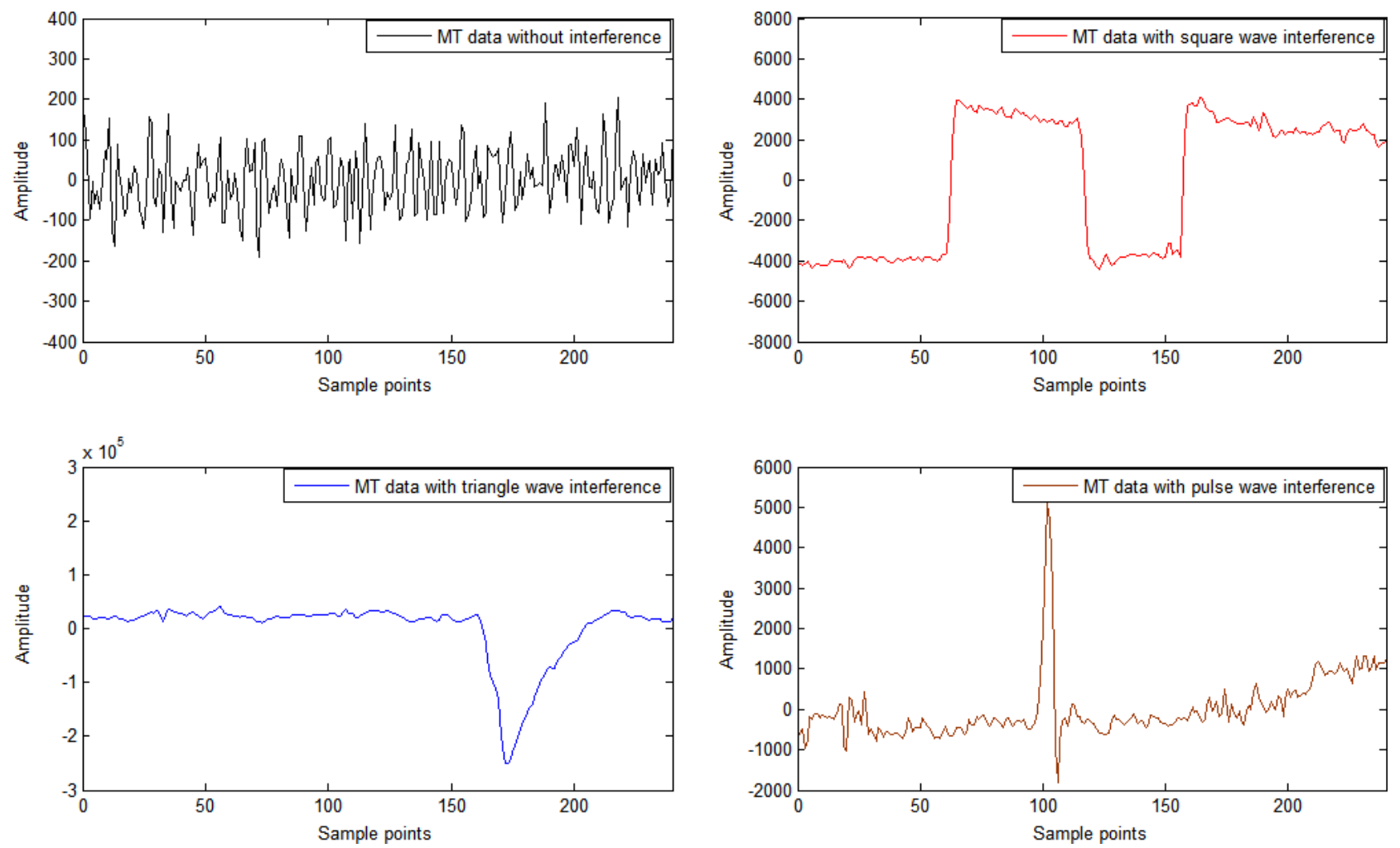

Fig. 1 MT data samples in library.

Figure 1 gives an example from each of the four types of the MT time-series data. From Fig. 1, the magnitude of MT data with strong interference is much larger than the MT data without interference, and the overall waveform dramatically varies.

In order to quantitatively characterize the useful signals and the strong interference, FBD, HFD, FuEn and ApEn are extracted from the four MT data samples shown in Fig. 1, and the results are given in Table 1.

As shown in Table1 all the characteristic parameters of the MT data without interference are larger than those of the noise-corrupted data. According to the physical meaning of fractal-entropy, the MT data without interference contains more randomness and complexity. Therefore, these characteristic parameters can more intuitively reflect the intrinsic characteristics of MT data.

\subsection{Clustering}

These four types of characteristic parameters are extracted from all the samples in the library and analyzed by FCM clustering. Figure 2 is the result of FCM clustering for the sample library.

From Fig. 2, the sample library is clearly divided into two categories by FCM clustering. All MT data without interference are classified as Type 1, and other MT data with strong interference are classified as Type 2. The results confirm that the fractalentropy characterization and the clustering algorithm are effective and robust to the data samples with known features.

\subsection{Targeted De-Noising}

Numerical datasets are constructed to validate automated signal-noise identification based on

Table 1 Characteristic Parameters of the Example MT Data Samples.

\begin{tabular}{lcccc}
\hline Type $\backslash$ Parameters & FBD & HFD & FuEn & ApEn \\
\hline MT data without interference & 1.4904 & 2.0066 & 1.6837 & 1.1289 \\
MT data with square wave interference & 1.4030 & 1.2336 & 0.4008 & 0.4779 \\
MT data with triangle wave interference & 1.1802 & 1.1599 & 0.1141 & 0.1569 \\
MT data with pulse wave interference & 1.3328 & 1.3216 & 0.5105 & 0.5083 \\
\hline
\end{tabular}




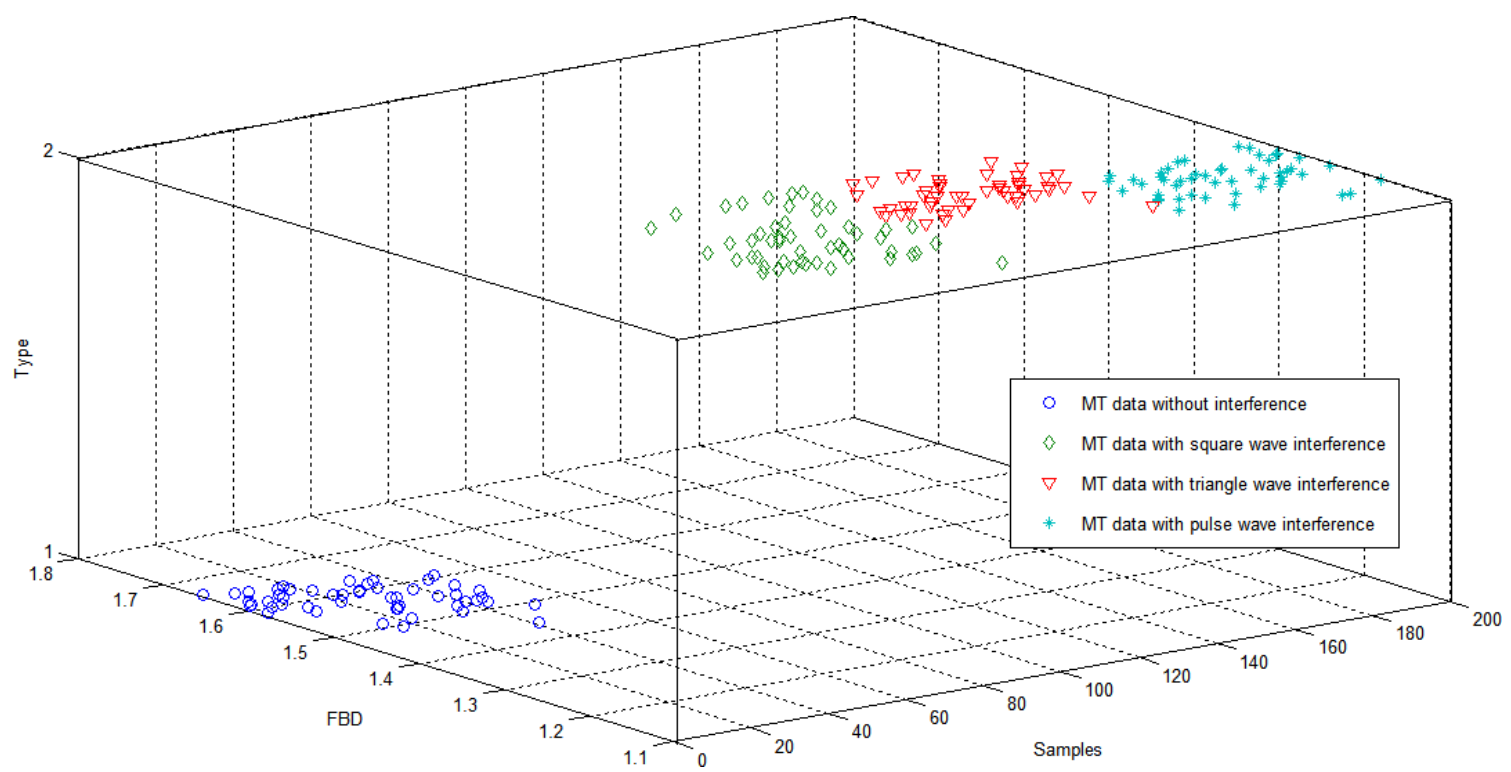

(a)

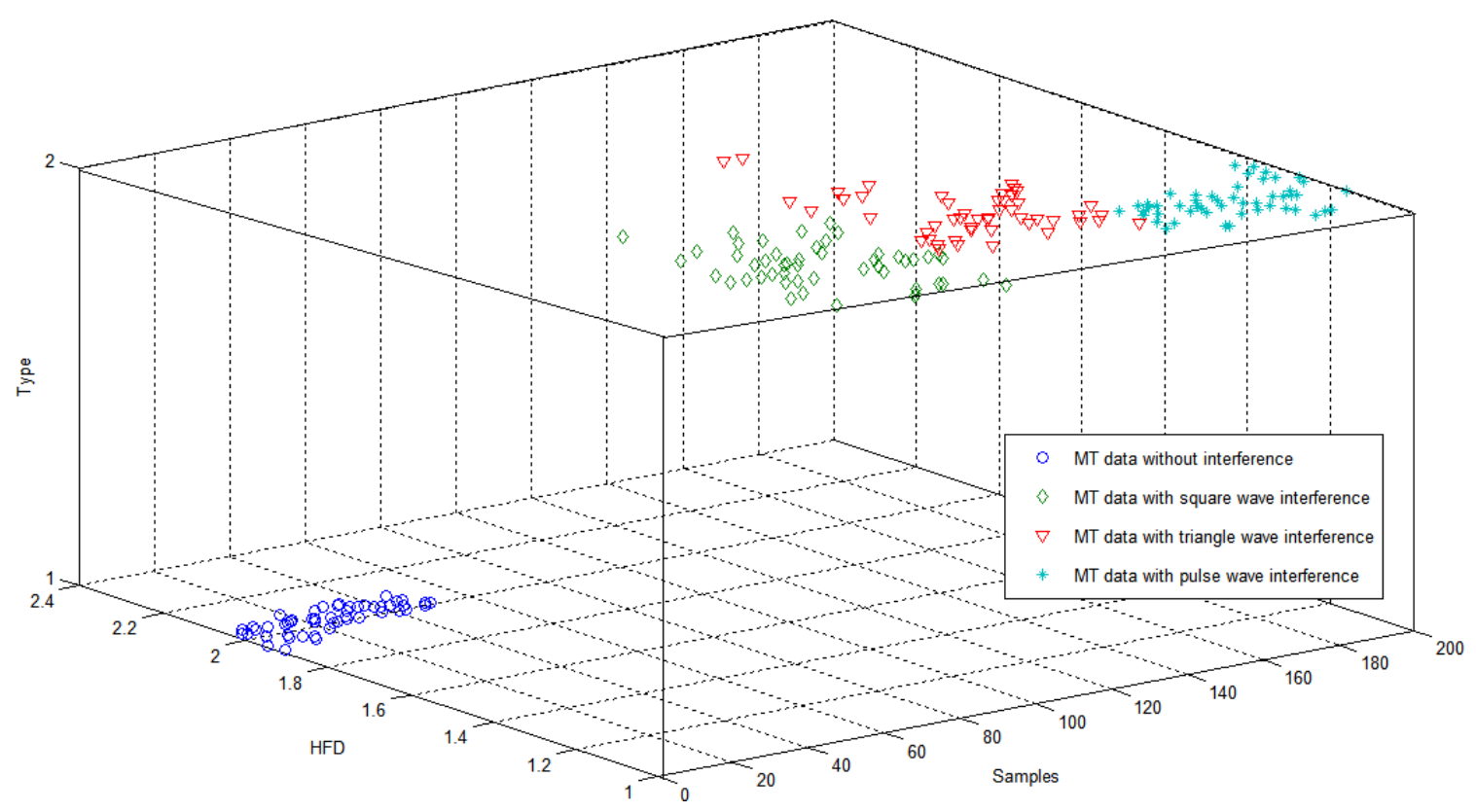

(b)

Fig. 2 Results of FCM clustering for the sample library, with the clustering analysis based on the characteristic parameter of (a) FBD; (b) HFD; (c) FuEn and (d) ApEn.

clustering and targeted de-noising using the WT technique. Square wave interference and triangle wave interference are imposed on white noise signals to construct the datasets. The previously discussed four characteristic parameters are extracted from the time-series. Then, FCM clustering is used to identify signal section with and without strong interference. WT de-noising method is used only to suppress the identified strong interference, and the filtered signal sections are connected with the unfiltered signal sections to reconstruct the whole signal. Figure 3 shows the process and the results.

The results in Fig. 3 confirm that the proposed method can accurately identify signal sections with strong interference in the time domain, and the targeted WT de-noising effectively removes the interference without compromising the useful information in signal sections with no interference. 


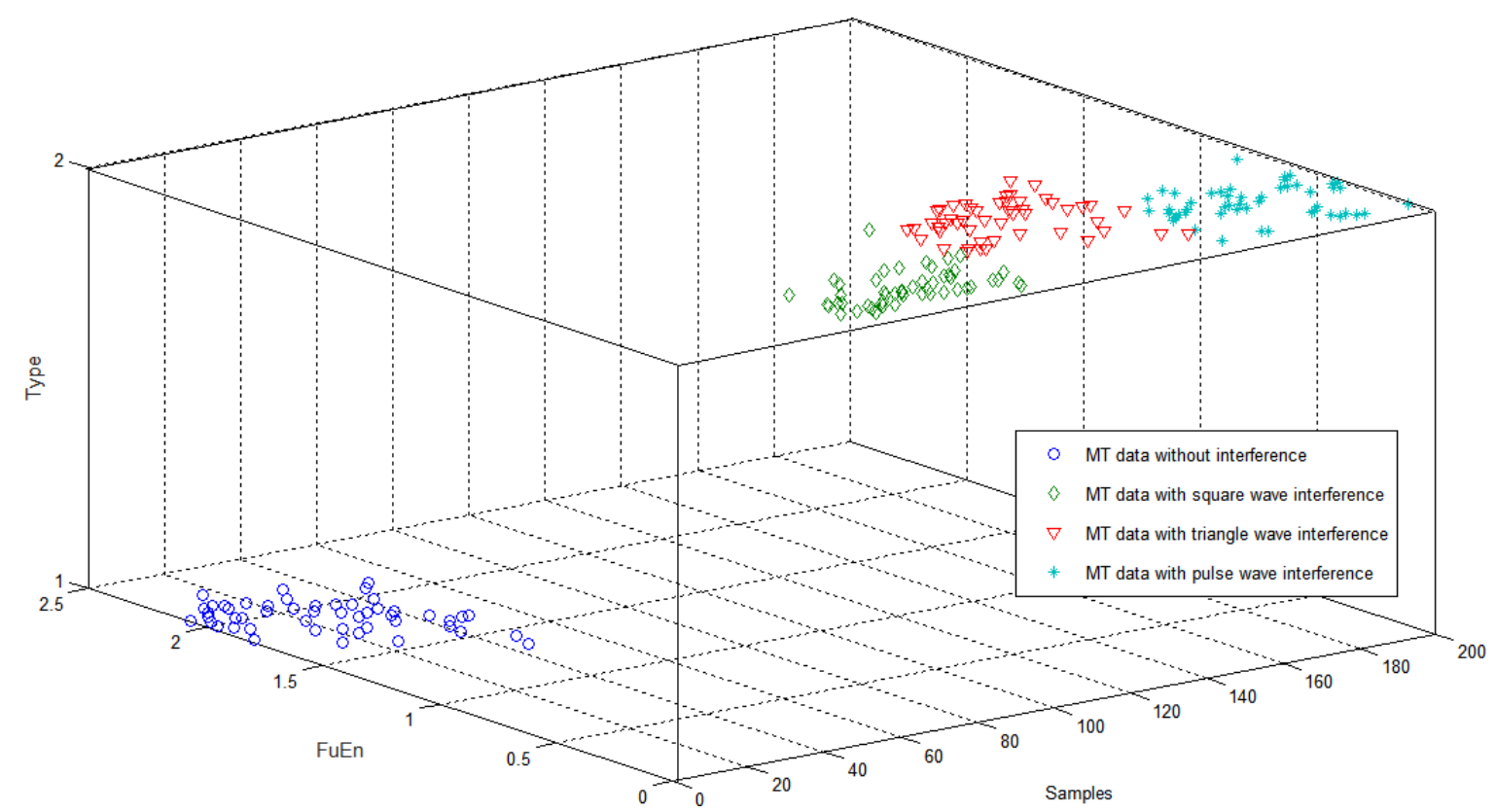

(c)

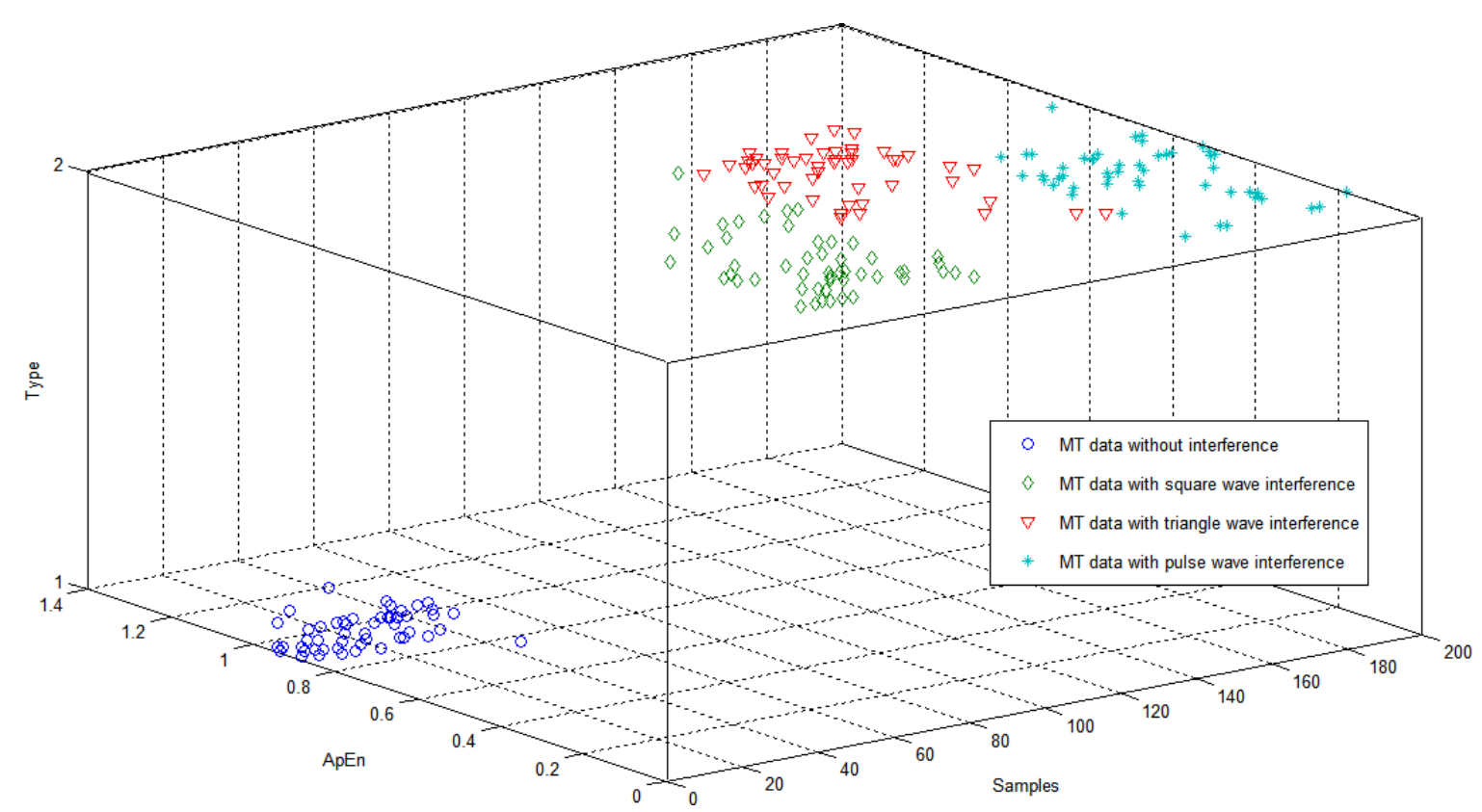

(d)

Fig. 2 (Continued)

\section{APPLICATION AND DISCUSSION}

\subsection{Signal Noise Identification and Targeted De-Noising for Measured Data}

In order to further verify the usefulness of the proposed method for field applications, three sets of measured MT data with typical strong interferences (square, triangle and pulse waves) are used for analysis. The measured data is collected by the V5-2000 instrument (Phoenix Geophysics, Toronto, Canada) from the Luzong ore concentration area.

Figure 4 shows the results of the automated signal-noise identification using the fractal-entropy and clustering technique. The identified signal 
Signal-Noise Identification of MT Signals
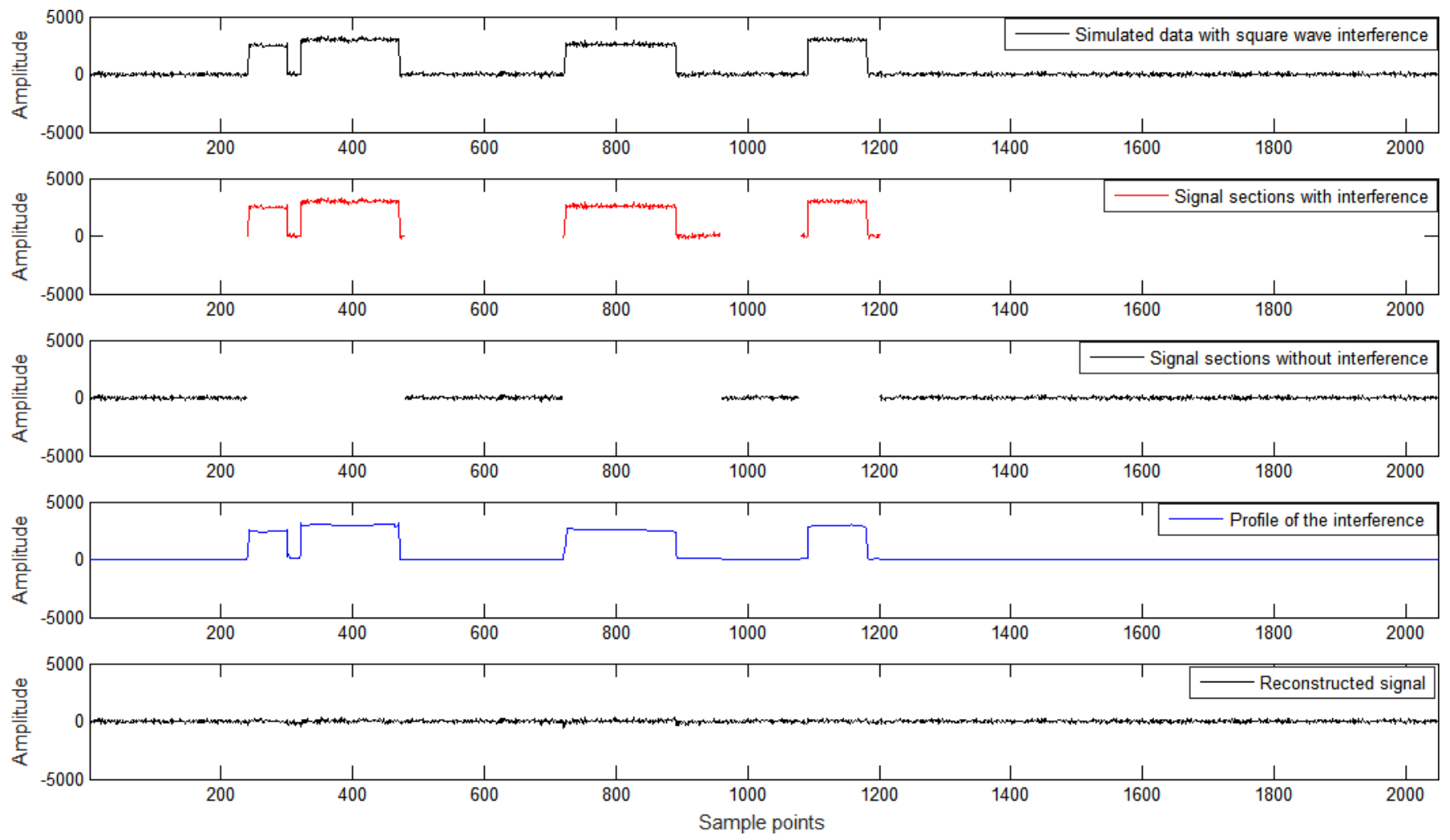

(a)
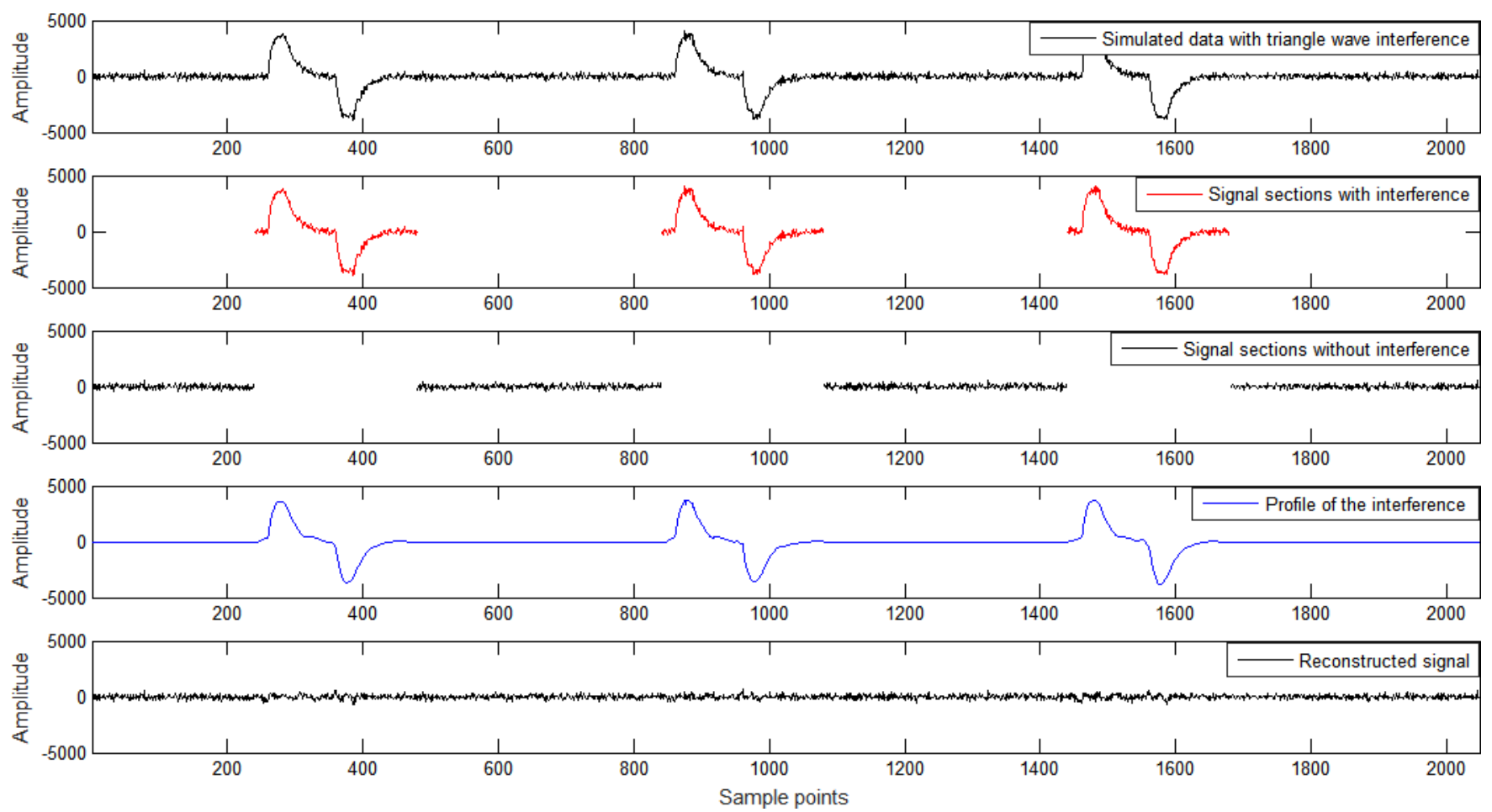

(b)

Fig. 3 Verification of signal-noise identification and targeted de-noising using simulated data: (a) separation for simulated data with square wave interference; and (b) with triangle wave interference. 

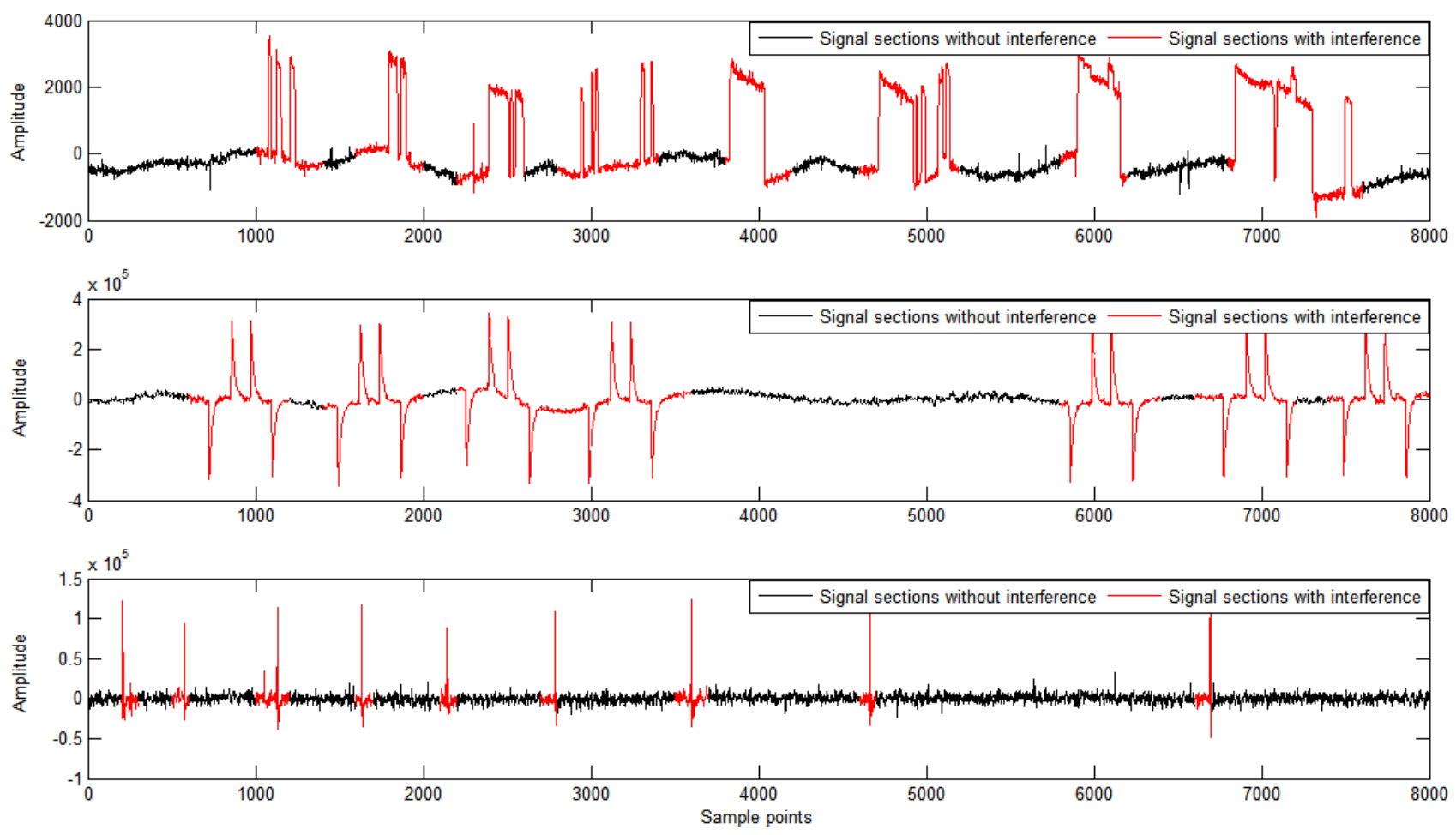

Fig. 4 Results of the automated signal-noise identification using the fractal-entropy and clustering technique for field measured MT data with square, triangle and pulse wave interference.

sections with interference are highlighted in red. The result confirms that the proposed method can effectively identify signal sections without interference and those with typical strong interferences.

The identified signal sections with interference are extracted and filtered by the WT technique, before putting back to reconstruct the whole signal. Figure 5 shows the process and results, with comparisons to the results from the conventional WT overall de-noising approach (i.e. the filter is applied to the whole signal).

From Fig. 5, due to the fact that the WT overall processing approach does not involve signal-noise identification, the slow-change components of lowfrequency are also filtered out together with the strong interference, resulting in all filtered MT signals close to the baseline. As the proposed method quantitatively identifies useful signal and strong interference, it purposefully suppresses interference, avoiding the blindness of the WT overall processing method, and reserving more slow-change components of low-frequency.

\subsection{Geophysical Analysis for the Measurement Site}

Geophysical analysis is undertaken on the original data, data filtered by the WT overall approach, and the data filtered by the proposed technique. Figure [6] shows the comparison of the apparent resistivity-phase curves for MT measurement site D36390.

The data was collected for about $20 \mathrm{~h}$ and the sampling frequency was divided into high-frequency $(2560 \mathrm{~Hz})$, intermediate-frequency $(320 \mathrm{~Hz})$ and low-frequency $(24 \mathrm{~Hz})$. The high-frequency data and intermediate-frequency data were alternately collected in the whole period, and stored in the TSH file format. The low-frequency data was collected in the whole period and stored in the TSL file format. Since most geophysical information about the measurement site is concentrated in the lowfrequency, only the TSL file is analyzed in detail. In Fig. 6a, the apparent resistivity curve of the original data is nearly $45^{\circ}$ asymptote rising between $50 \mathrm{~Hz}$ and $0.05 \mathrm{~Hz}$, and the corresponding phase is $0^{\circ}$ or $180^{\circ}$. About 15 frequency points are completely distorted, and the value of the apparent resistivity even exceeds $100,000 \Omega \cdot \mathrm{m}$, which is unlikely to be realistic. In the $0.05-0.005 \mathrm{~Hz}$ frequency band, the value of the apparent resistivity suddenly drops to $100 \Omega \cdot \mathrm{m}$. This phenomenon is resulted from the typical near-source effect, and the result does not truly reflect the underground geoelectric information. 

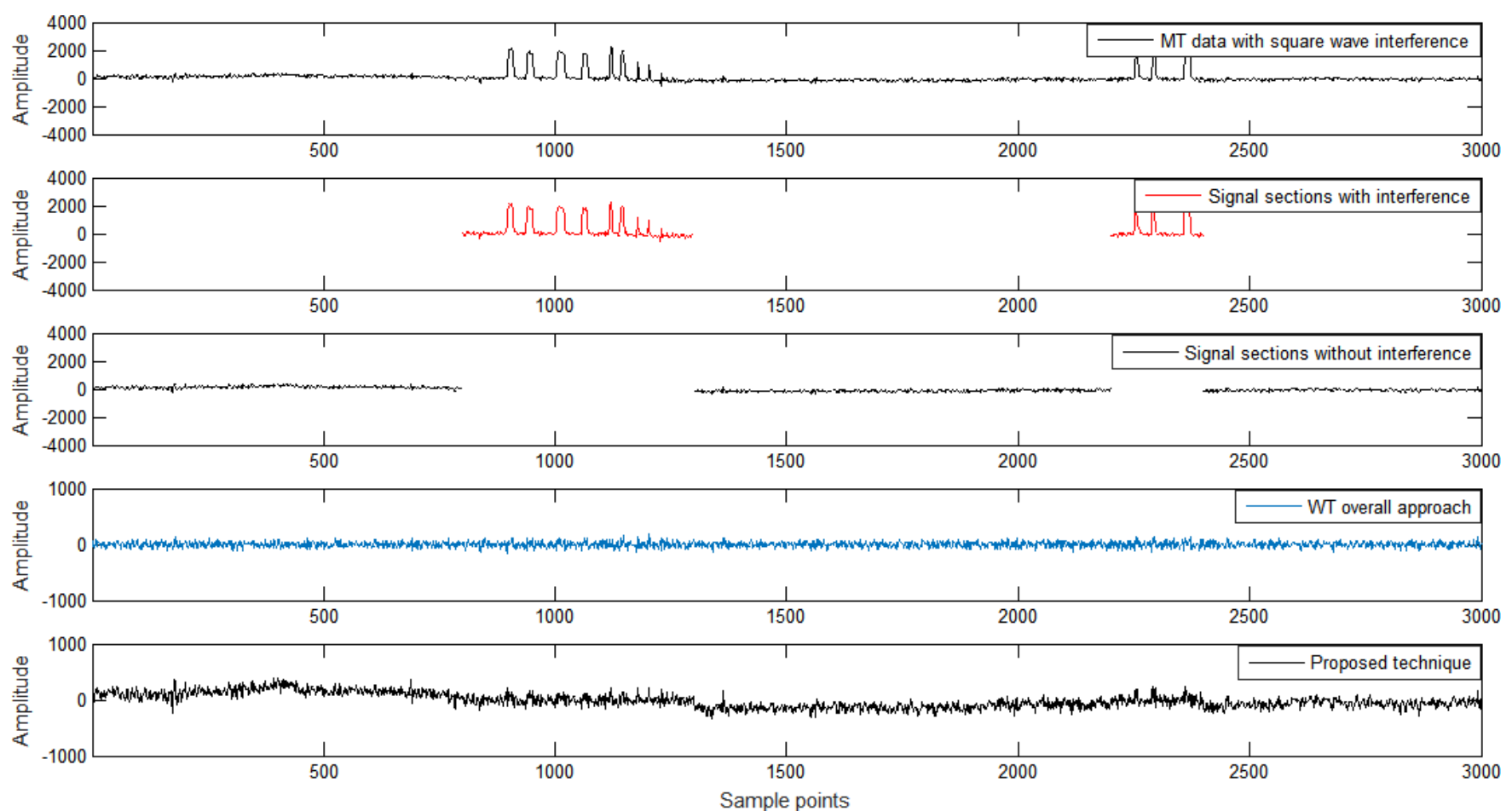

(a)
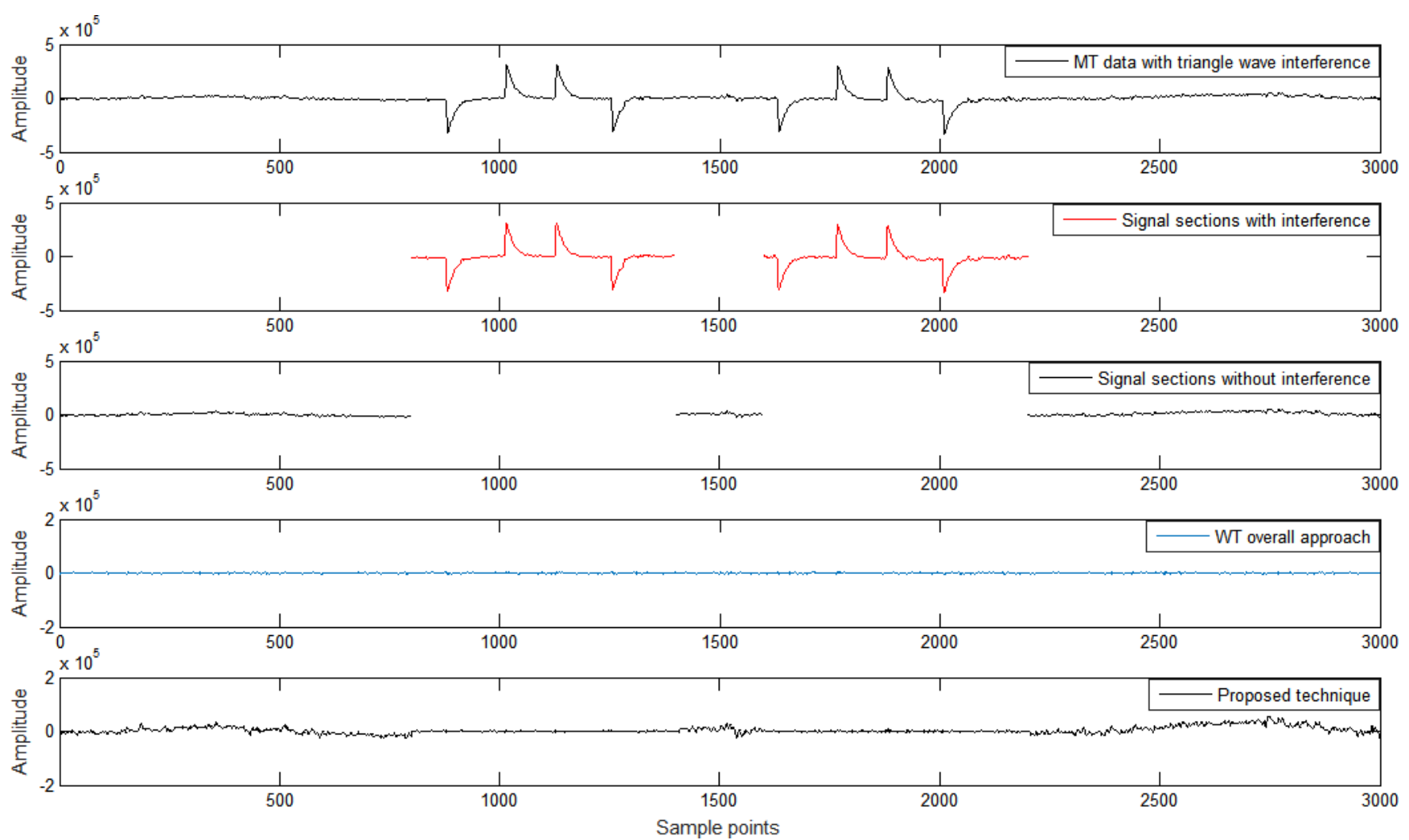

(b)

Fig. 5 The process and results of targeted de-noising for field measured MT data, with comparisons to the results from the conventional WT overall de-noising approach: (a) targeted de-noising of square wave interference for measured MT data; and (b) targeted de-noising of triangle wave interference for measured MT data. 
J. Li et al.
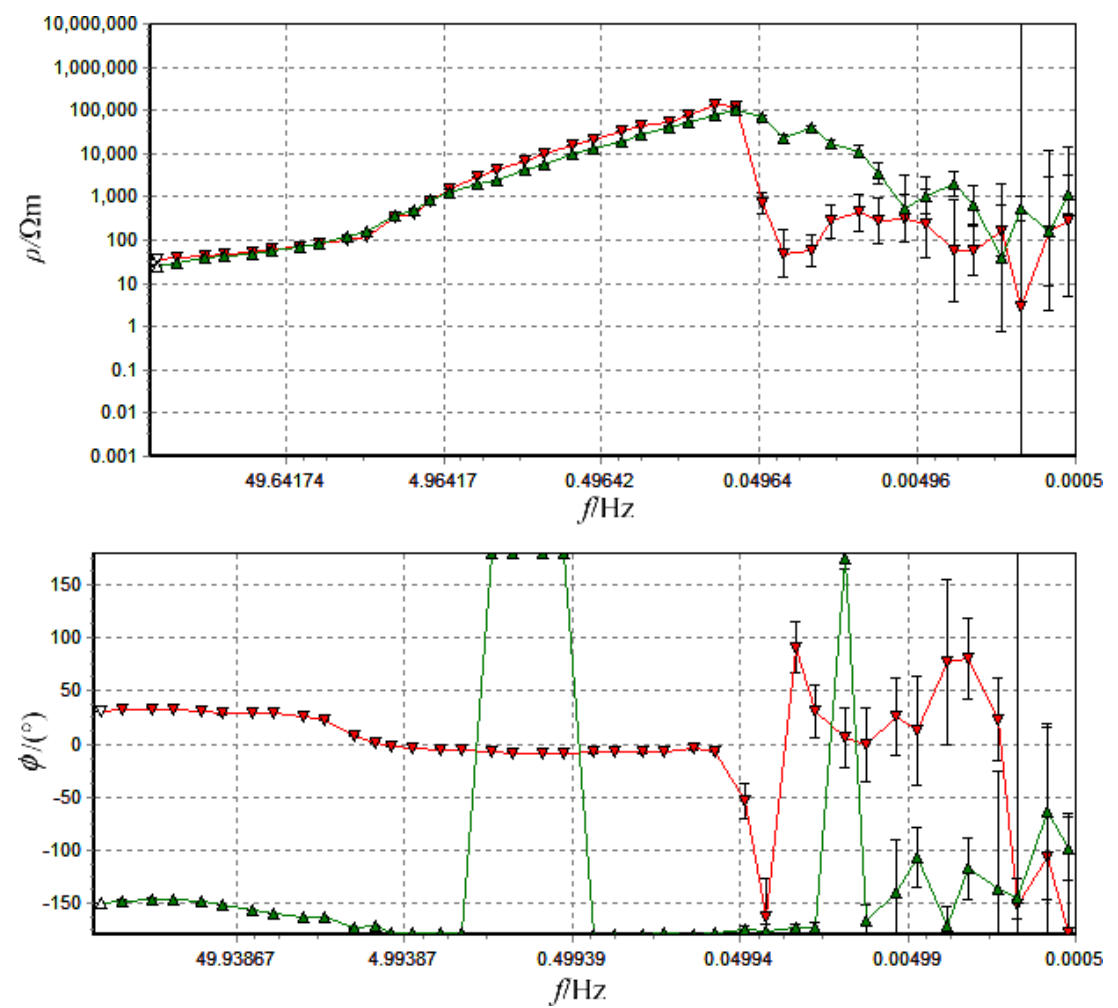

(a)
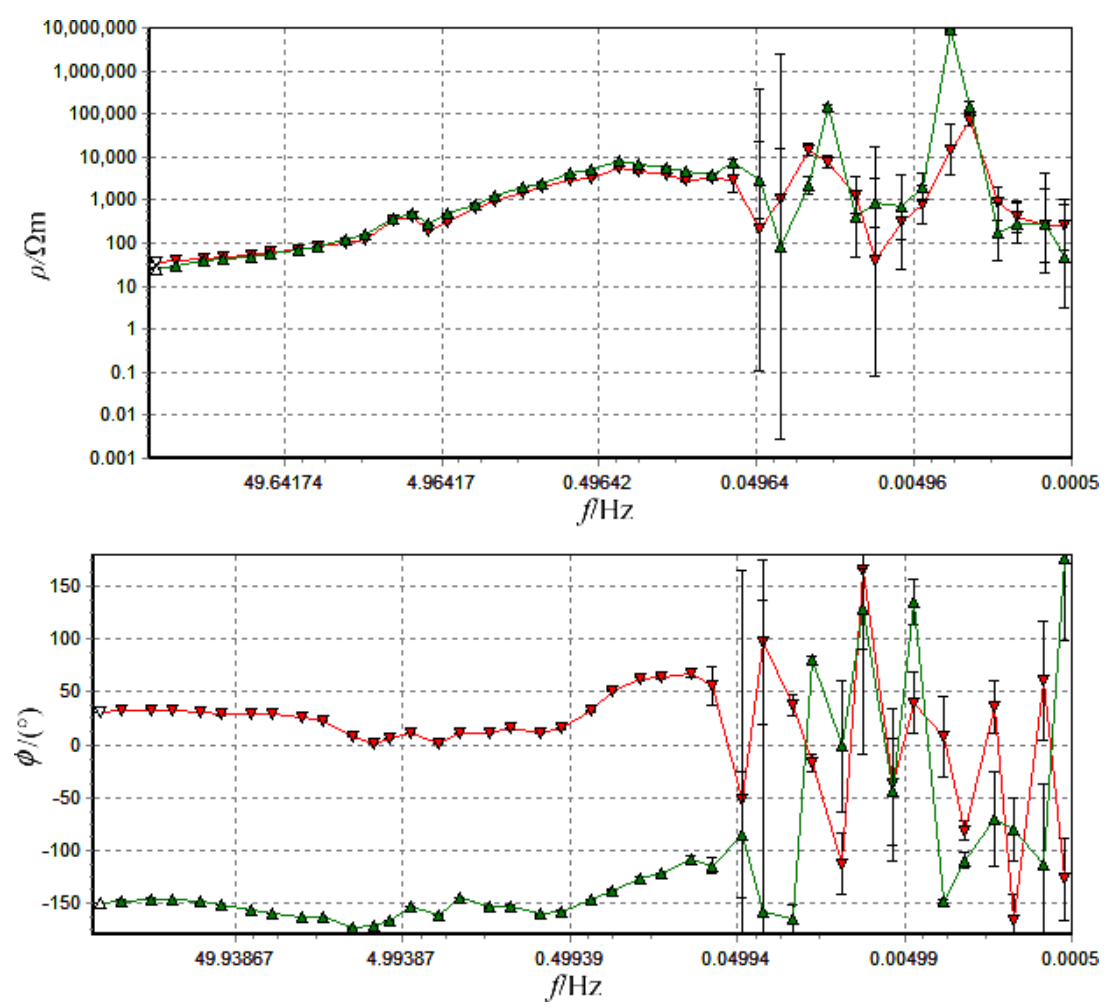

(b)

Fig. 6 Comparison of the apparent resistivity-phase curves for MT measurement site D36390, as derived from: (a) original data; (b) data filtered by WT overall approach; and (c) data filtered by the proposed technique. 

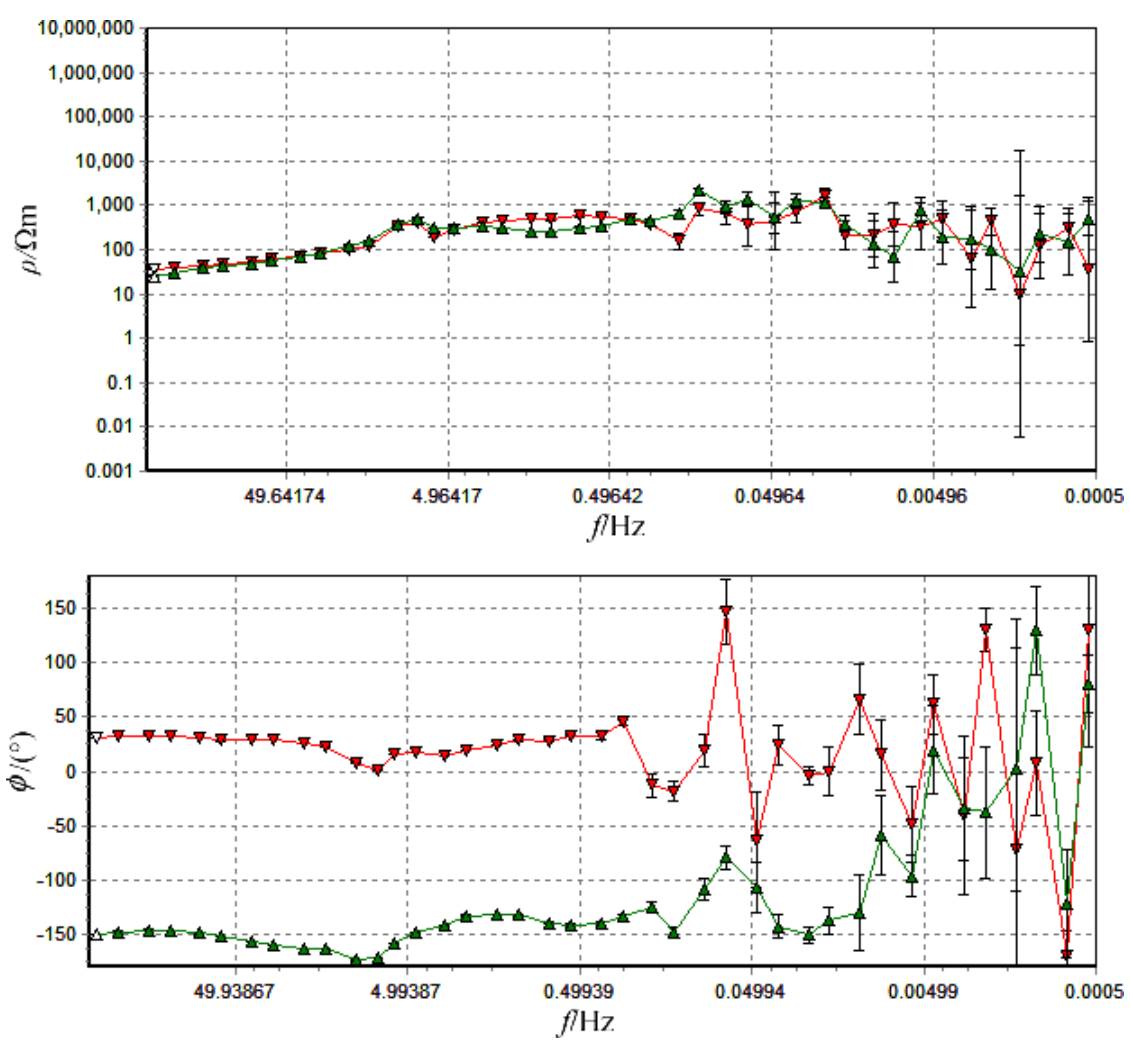

(c)

Fig. 6 (Continued)

Figure 6 $6 \mathrm{~b}$ is the apparent resistivity-phase curve obtained from the data filtered by the WT overall processing method. In the $0.1-0.0005 \mathrm{~Hz}$ frequency band, the apparent resistivity curve has significant oscillations, and the phase curve is also disturbed. At the same time, the corresponding error bars are significant. Although the WT overall processing method can suppress the large-scale strong interference, the slow-change components of low-frequency have also been filtered out. The result still cannot effectively reflect the underground geoelectric information.

Figure 6re is the apparent resistivity-phase curve obtained from the data processed by the proposed technique. As the proposed technique includes signal-noise identification, it is only needed to suppress the identified strong interference. From Fig. 6c, the overall apparent resistivity curve is more smooth and continuous, and the error bars are generally moderate. Moreover, the value of the apparent resistivity is relatively stable, and the corresponding phase is improved obviously. Compared with the WT overall processing method, the result of the proposed technique retains more useful MT information.
Figure 7 is the comparison of the apparent resistivity-phase curve for AMT measurement site D012455.

The data was collected for about $1 \mathrm{~h}$ and the sampling frequency was divided into high-frequency $(24,000 \mathrm{~Hz})$, intermediate-frequency $(2400 \mathrm{~Hz})$ and low-frequency $(150 \mathrm{~Hz})$. The high-frequency data and intermediate-frequency data were alternately collected in the whole period, and stored in TS2 and TS3 format, respectively. The low-frequency data is collected in the whole period and stored in TS4 format. From the time trace of the original data (not shown in this paper), it can be seen that the original data is disturbed by triangle wave interference and pulse wave interference significantly. From Fig. 7a, the apparent resistivity-phase curve derived from the original data has significant oscillations in the $10-0.3 \mathrm{~Hz}$ frequency band, and the corresponding error bars increase with the decrease in frequency significantly. According to the above analysis, the original data of measured AMT site D012455 cannot reasonably reflect the underground geoelectric information.

Figure 7b is the apparent resistivity-phase curve obtained from the data filtered by the WT over- 
J. Li et al.
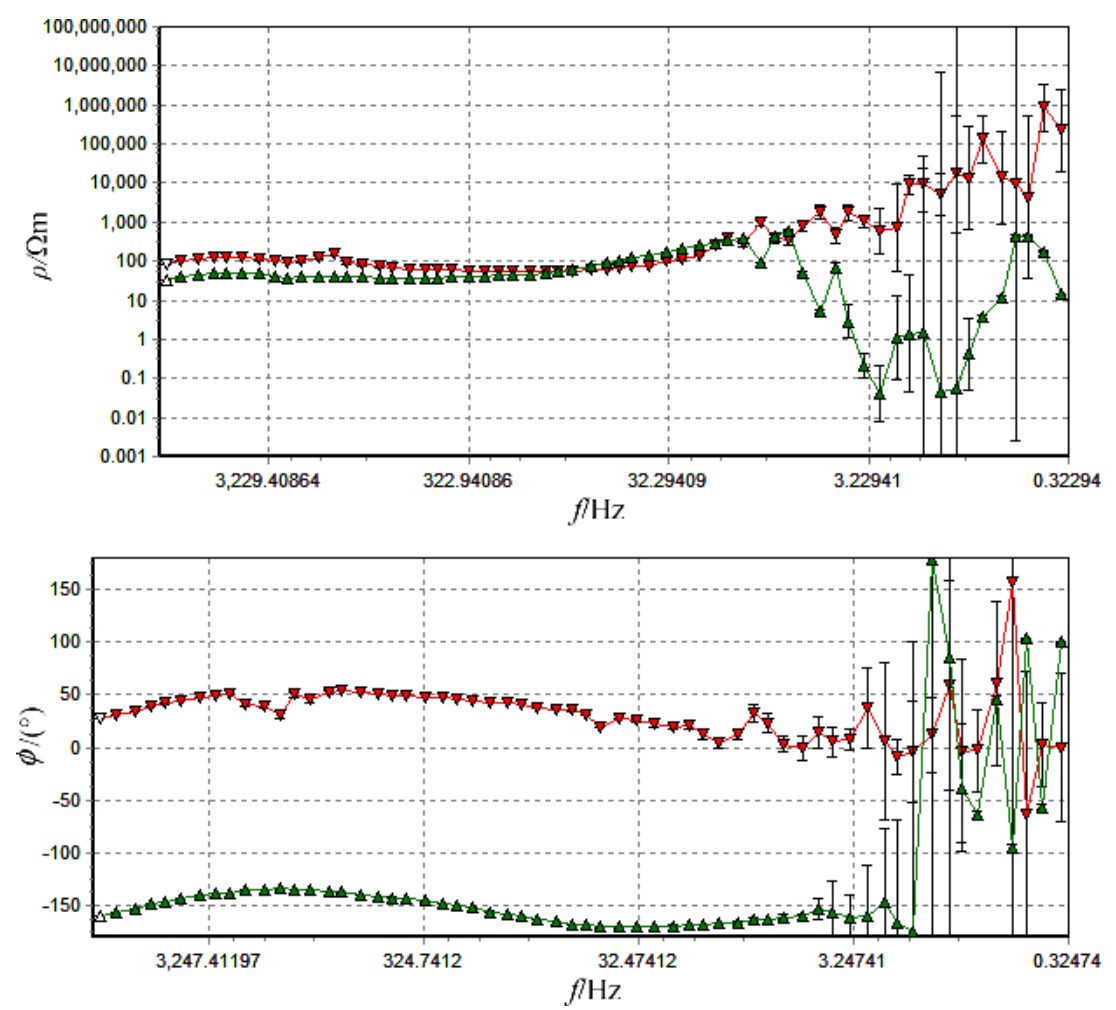

(a)
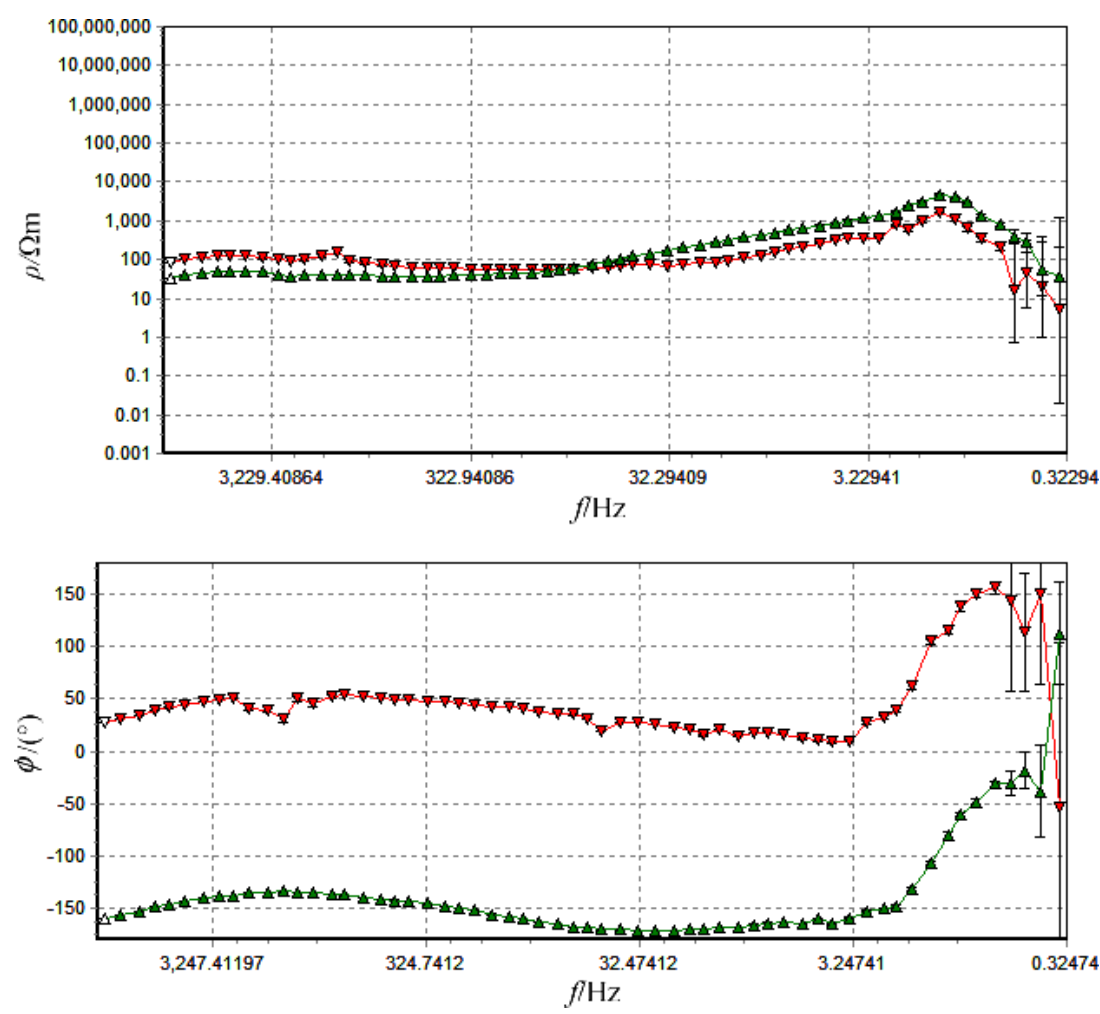

(b)

Fig. 7 Comparison of the apparent resistivity-phase curves for AMT measurement site D012455, as derived from: (a) original data; (b) data filtered by WT overall approach; and (c) data filtered by the proposed technique. 

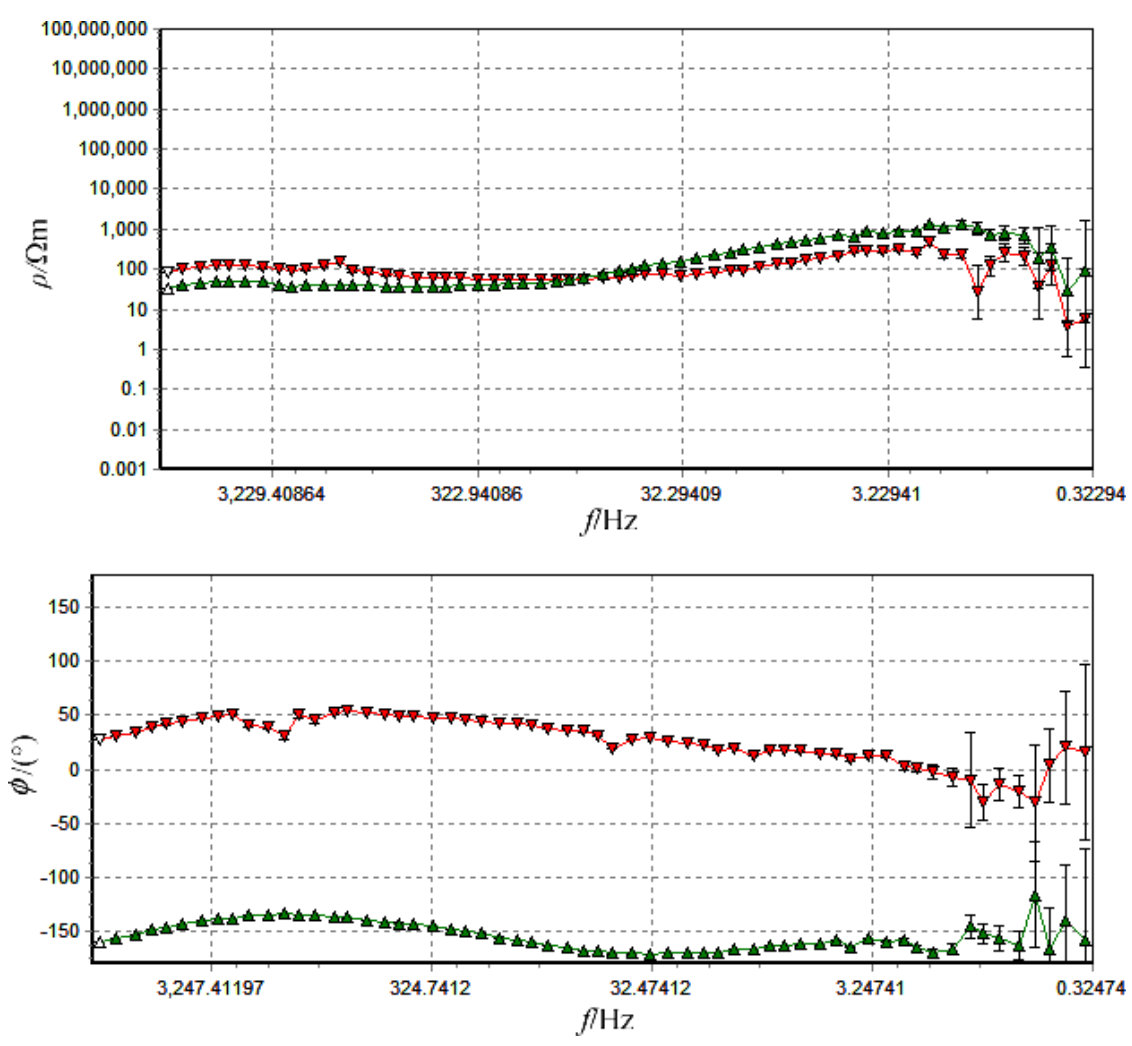

(c)

Fig. 7 (Continued)

all processing method. From Fig. 7b, the apparent resistivity curve has a downward trend in the $3.2-0.32 \mathrm{~Hz}$ frequency band, and the corresponding phase curve has significant oscillations and error bars increase significantly.

Figure $7 \mathrm{r}$ is the apparent resistivity-phase curve obtained from the data processed by the proposed method. From Fig. 77c, the apparent resistivityphase curve is raised obviously in the $3.2-0.32 \mathrm{~Hz}$ frequency band when compared with Fig. $7 \mathrm{~b}$, and the values of the apparent resistivity are within a reasonable range. The whole curve becomes smooth and continuous, and the error bars are decreased significantly.

In order to further verify the effectiveness of the proposed technique, the polarization direction of electromagnetic field is calculated and analyzed. Figure 8 is the comparison of the polarization direction results (at $0.5 \mathrm{~Hz}$ ) as derived from the original data and from the data processed by the proposed technique for AMT measurement site D012455.

According to the definition of polarization direction of the electromagnetic field, the natural field is usually motivated by a variety of sources, and the polarization direction changes randomly with time. If there is a strong electromagnetic interference, the polarization direction of electromagnetic field may be dominated by these active sources and intensively concentrated on a certain direction, which results in a focus directionality. As shown in Fig. 8, the polarization direction of electric channel as derived from the original data is concentrated between $-90^{\circ}$ and $-40^{\circ}$, and the polarization direction of magnetic channel from the original data is concentrated between $-20^{\circ}$ and $20^{\circ}$. The reason for the focused polarization direction is that the original data is disturbed by different types of strong electromagnetic interference. By analyzing the data processed by the proposed technique, the polarization directions of the electric channel and the magnetic channel are scattered in different directions, which is close to the random characteristics of natural field. Combined with the apparent resistivity-phase curve and the polarization direction of electromagnetic field, it has been confirmed that the proposed technique can effectively suppress the strong interference in the Luzong ore concentration area and the reconstructed MT/AMT signal can more accurately reflect the underground geoelectric information. 

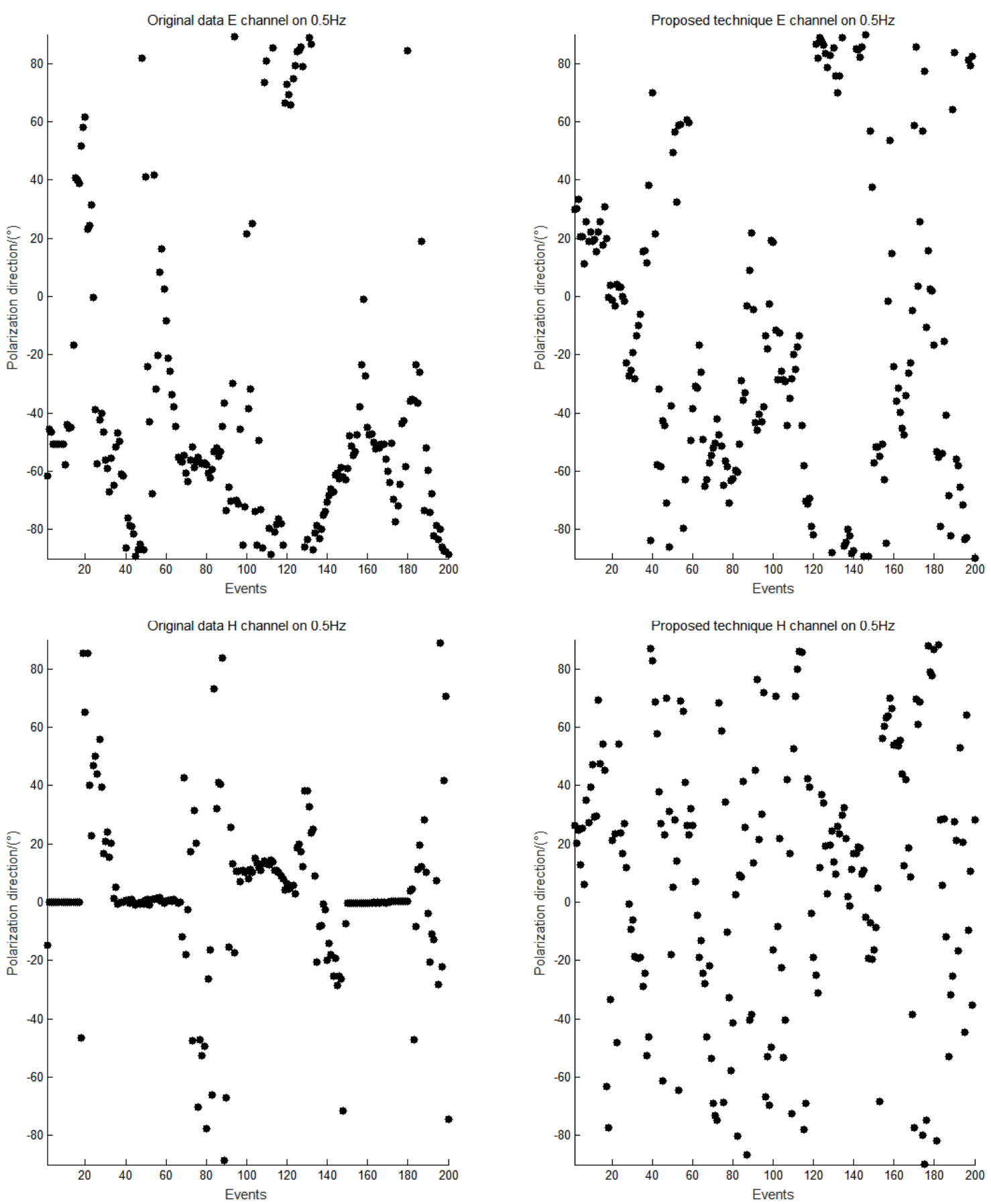

(a)

(b)

Fig. 8 Comparison of the polarization direction results for AMT measurement site D012455, derived from original data (a) and data filtered by the proposed technique (b).

\section{CONCLUSIONS}

A new technique for MT/AMT data pre-processing has been developed. The technique conducts automated signal-noise identification using fractalentropy analysis and FCM clustering characterization, before applying the WT based de-noising only to the signal sections identified as with strong interference. The signal-noise identification process enables targeted de-noising, significantly reduces the loss of useful information when compared with conventional pre-processing techniques that apply noise filters to the whole data.

The proposed technique has been verified by a library of data samples with known interference 
and simulated datasets with added interference. The results show that the four characteristic parameters - FBD, HFD, FuEn and ApEn — are suitable to describe the different features of signals sections with and without common interference (square, triangle and pulse waves), and the FCM clustering can robustly and automatically differentiate the two types of signals based on the four characteristic parameters. The targeted de-noising enables the slow-change components of low-frequencies be reserved, which enhances the subsequent geophysical analysis since the most useful information is included in the low-frequency components.

The usefulness of the proposed technique has been further verified by field measured MT/AMT data. Compared with the corresponding results from the original data and the data filtered by the WT overall approach, the apparent resistivityphase curve resulted from the data processed by the proposed technique is more smooth and continuous, and the result more realistically reflects the inherent underground geoelectric structure information of the MT/AMT measurement site itself.

\section{ACKNOWLEDGMENTS}

This work is supported by the National Natural Science Foundation of China (41404111) and the National High Technology Research and Development Program of China (2014AA06A602).

\section{REFERENCES}

1. N. Rees et al., Magnetotelluric monitoring of coalseam gas and shale-gas resource development in Australia, Lead. Edge 35(1) (2016) 64-70.

2. G. D. Egbert, Robust multiple-station magnetotelluric data processing, Geophys. J. Int 130(2) (1997) 475-496.

3. Z. Y. Ren et al., A finite-element-based domaindecomposition approach for plane wave 3D electromagnetic modeling, Geophysics 79(6) (2014) E255E268.

4. J. H. Cai et al., An analysis method for magnetotelluric data based on the Hilbert-Huang transform, Explor. Geophys. 40(2) (2009) 197-205.

5. K. N. Kappler, A data variance technique for automated despiking of magnetotelluric data with a remote reference, Geophys. Prospect. 60(1) (2012) 179-191.
6. J. T. Tang et al., Mathematical morphology filtering and noise suppression of magnetotelluric sounding data, Chinese J. Geophys. 55(5) (2012) 17841793.

7. J. Li et al., Noise suppression for magnetotelluric sounding data based on signal subspace enhancement and endpoint detection, Acta Phys. Sin. 63(1) (2014) 019101.

8. D. O. Trad and J. M. Travassos, Wavelet filtering of magnetotelluric data, Geophysics 65(2) (2000) 482491.

9. J. C. Cai et al., Electrical conductivity models in saturated porous media: A review, Earth-Sci. Rev. 171 (2017) 419-433.

10. J. Lévy-Véhel, Fractal approaches in signal processing, Fractals 3(4) (1995) 755-775.

11. R. R. Coifman and M. V. Wickerhauser, Entropybased algorithms for best basis selection, IEEE Trans. Inform. Theory 38(2) (1992) 713-718.

12. B. Pesquet-Popescu and J. Lévy-Véhel, Stochastic fractal models for image processing, IEEE Signal Proc. Mag. 19(5) (2002) 48-62.

13. A. P. Singh et al., Seismic source characteristics in kachchh and saurashtra regions of western india: bvalue and fractal dimension mapping of aftershock sequences, Nat. Hazards 77(S1) (2015) 33-49.

14. J. C. Cai et al., Fractal characterization of dynamic fracture network extension in porous media, Fractals 25(2) (2017) 1750023.

15. W. Wei et al., An electrical conductivity model for fractal porous media, Geophys. Res. Lett. 42(12) (2015) 4833-4840.

16. B. M. Yu and J. H. Li, Fractal dimensions for unsaturated porous media, Fractals 12(1) (2004) 17-22.

17. J. Li et al., Magnetotelluric noise suppression base on signal-to-noise identification in ore concentration area, Chinese J. Geophys. 60(2) (2017) 722-737.

18. J. J. Sun and Y. G. Li, Joint inversion of multiple geophysical and petrophysical data using generalized fuzzy clustering algorithms, Geophys. J. Int 208(2) (2017) 1201-1216.

19. J. Xu et al., Acoustic-based cutting pattern recognition for shearer through fuzzy c-means and a hybrid optimization algorithm, Appl. Sci. 6(10) (2016) 294311.

20. S. Abbasion et al., Rolling element bearings multifault classification based on the wavelet denoising and support vector machine, Mech. Syst. Signal Pr. 21(7) (2007) 2933-2945.

21. N. Sarkar and B. B. Chaudhuri, An efficient differential box-counting approach to compute fractal dimension of image, IEEE Trans. Syst. Man Cybern. 24(1) (1994) 115-120.

22. M. N. Akhtar, M. G. P. Prasad and M. A. Navascués, Box dimension of $\alpha$-fractal function, Fractals 24(3) (2016) 1650037. 
23. L. S. Liebovitch and T. Toth, A fast algorithm to determine fractal dimensions by box counting, Phys. Lett. A 141(8-9) (1989) 386-390.

24. T. Higuchi, Approach to an irregular time series on the basis of the fractal theory, Physica D 31(2) (1988) 277-283.

25. S. Spasic et al., Different anesthesia in rat induces distinct inter-structure brain dynamic detected by higuchi fractal dimension, Fractals 19(1) (2011) $113-123$.

26. A. Accardo et al., Use of the fractal dimension for the analysis of electroencephalographic time series, Biol. Cybern. 77(5) (1997) 339-350.

27. S. Spasić et al., Estimation of parameter $k_{\max }$ in fractal analysis of rat brain activity, Ann. NY Acad. Sci. 1048 (2005) 427-429.
28. B. Kosko, Fuzzy entropy and conditioning, Inform. Sciences 40(2) (1986) 165-174.

29. S. Pincus, Approximate entropy (ApEn) as a complexity measure, Chaos 5(1) (1995) 110-117.

30. J. Z. Wang et al., A modified FCM algorithm for MRI brain image segmentation using both local and non-local spatial constraints, Comput. Med. Imag. Grap. 32(8) (2008) 685-698.

31. J. O. Deasy, M. V. Wickerhauser and M. Picard, Accelerating monte carlo simulations of radiation therapy dose distributions using wavelet threshold de-noising, Med. Phys. 29(10) (2002) 2366-2373. 